\title{
Human Movement Analysis: Extension of the F-Statistic to Time Series using HMM
}

\author{
Michelle Karg ${ }^{1}$, Wolfgang Seiberl ${ }^{2}$, Jesse Hoey ${ }^{3}$, Dana Kulić ${ }^{1}$ \\ ${ }^{1}$ Department of Electrical and Computer Engineering, University of Waterloo, Waterloo, Canada \\ ${ }^{2}$ Department of Biomechanics in Sports, Technische Universität München, Munich, Germany \\ ${ }^{3}$ Cheriton School of Computer Science, University of Waterloo, Waterloo, Canada
}

\begin{abstract}
Optical motion tracking has enhanced human movement analysis in medicine, biomechanics, and rehabilitation science by providing highly accurate joint angle measurements over time. However, analyzing the large amount of recorded data is challenging. The process is usually simplified by calculating descriptive measures, such as the minimum, mean, or maximum, from the time series data. We propose a novel technique for the analysis of human motion data, which considers the complete time series data and is based on the F-statistic traditionally used in medical and biomechanical studies. The time series data is modeled by a Hidden Markov Model (HMM) and the Fstatistic is reformulated using the Kullback-Leibler divergence for comparing HMMs. This provides a novel technique to enhance the analysis of human movement data and includes an automatic generation of group-specific trajectories to simplify visual data analysis. It is further suitable as time-series based, univariate feature selection technique in machine learning.
\end{abstract}

Index Terms-Hidden Markov Model, F-Statistic, Human Movement Analysis, Biomechanics.

\section{INTRODUCTION}

Human movement analysis is a key element in biomechanics. It is used to investigate the influence of factors, such as individual characteristics or chronic diseases on the human kinematics, to qualify the efficiency of treatments or surgeries, and to develop exercise strategies which are most effective [1], [2], [3]. The outcome is beneficial to find better treatments and more suitable rehabilitation exercises for populations suffering from chronic diseases or after injuries or surgeries, to optimize the training of athletes, and to enhance the fitness of the healthy population. Furthermore, investigating the kinematics of the healthy population provides a systematic way to compare the efficiency and safety of different movement strategies for developing guidelines to avoid movement strategies which are less suitable or more prone to injuries [4].

Optical motion capture is a technology to record human movements and to reconstruct the joint angles, joint center positions, velocities and accelerations with high accuracy. It is widely used in clinical and biomechanical studies to record gait, daily movements, and exercise movements of healthy subjects, athletes, or subjects suffering from chronic diseases, after injuries, or operations [1], [2], [3]. The data is usually recorded with a frame rate of up to $512 \mathrm{~Hz}$. This provides highly accurate estimates of the kinematic parameters during exercises, but also results in a large dimensionality of the recorded data set. Analyzing this amount of data with traditional methods is a challenge for physicians and

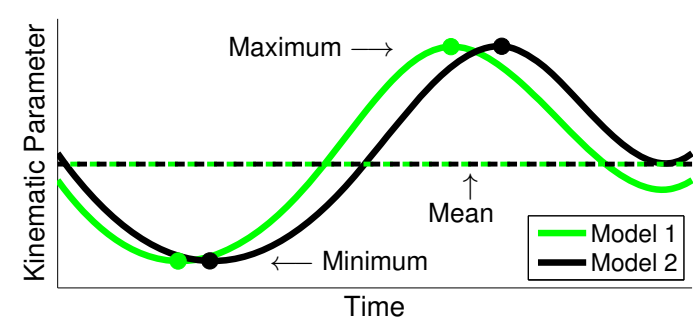

Figure 1. The descriptive measures minimum, mean, maximum, and ROM are the same for the exemplified time series of two motions (green and black). Calculating the traditional F-statistic to time series data generated by these two motions, would indicate that the two motions are the same. The HMM-based extension of the F-statistic to time series covers the illustrated temporal and spatial shifts of the two motions without the requirement to explicitly specify them and can successfully distinguish these two motions.

researchers in biomechanics. Usually, the time series data is visually analyzed for several joint angles, a small set of kinematic parameters are pre-selected, descriptive measures such as minimum, mean, maximum, or range of motion (ROM) of the time series data of these parameters are calculated, and a t-test or ANOVA (analysis of variance) are applied [5]. However, manual selection of the kinematic parameters and descriptive measures can be challenging, because movement execution differs between individuals and the selected set might not be the most informative. This can be overcome by utilizing computational techniques applied to the time series data to select the most informative movement kinematics [6], [7], [2], [8], [3].

We propose a novel method which extends the F-statistic to time series data. The time series of a kinematic parameter is modeled by a Hidden Markov Model (HMM) and the distance measures of the F-statistic are formulated as the Kullback-Leibler divergence (KL). The KL divergence has been widely applied to calculate the similarity of two HMMs. This novel technique automatically ranks the kinematic parameters according to differences in the time series without specifying descriptive measures. It overcomes the limitation of the traditional F-statistic which is based on individual descriptive measures. Changes in kinematic parameters can be revealed, which may be overseen by visual analysis or are not modeled by the selected descriptive measures.

The proposed approach is beneficial for physicians and researchers in biomechanics by providing automatic support in 
the selection of the kinematic parameters and for engineers and computer scientist as feature selection technique for developing machine learning algorithms, which discriminate different movement types or strategies.

The outline of the paper is as follows: Section II summarizes the state of the art on automatic human movement analysis for motion capture data. Section III introduces the HMM-based extension of the F-statisic. The utility of the proposed approach is investigated on a gait database and results are summarized in section IV. The paper concludes with a discussion in section $\mathrm{V}$ and a conclusion in section VI.

\section{Automated Human Movement Analysis}

Automatic human movement analysis is a promising approach in human movement science to overcome the challenges to quantify accurately and objectively human movements and to analyze large amounts of data. The approaches focus on developing sensor technologies, such as optical or inertial motion tracking, and developing machine learning algorithms which analyze movement data. The latter is most widely studied for gait analysis [2], [1], [6], [7]. In contrast to human motion analysis for action recognition or gait identification using computer vision [9], [10], databases in medicine and biomechanics are usually small and interpretability of the results plays an important role for clinical acceptance.

The majority of studies classify movements into a set of categories, which are e.g., normal or pathological movements, gender, different movement strategies, or emotions [1], [11], [4], [12]. These studies combine feature selection and classification. Computational intelligence (CI) techniques such as artificial neural networks, support vector machines, and Fuzzy rules are most widely used for classification besides statistical methods [8], [2]. Out of the overall set of measured kinematic parameters, a subset of features that achieves the highest recognition rate for the chosen classifier is selected. The most suitable feature set differs depending on the choice of the classifier. Lai et al. reason that the uptake of automatic human movement analysis in clinical applications has been slow because the relationship between factors and biomechanical observations is not easily and intuitively understandable from the outcome of CI techniques [2]. To achieve clinical acceptance, it is important that physicians and researchers in biomechanics can understand and also graphically visualize the relationship between factors and biomechanical changes in human movement. Besides techniques which provide as output the membership to movement categories, a ranking of the kinematic parameters is needed which is independent of the classification approach and generally understandable.

Human motion data is characterized by high dimensionality, typically requiring dimensionality reduction prior to classification. Dimensionality reduction can be subdivided in feature selection, projection, and compression techniques [13], where feature selection techniques have the advantage that they provide results that are easier to interpret because they do not alter the parameter space and select only a subset of them. Dimensionality reduction is challenging for high- dimensional human movement databases because of correlations, temporal dependencies, and nonlinear relationships between kinematic parameters, and high within-subject and between-subject variability [6], [7]. Common dimensionality reduction techniques, such as principal component analysis or the F-statistic for feature selection, cannot be directly applied to time series data. Therefore, descriptive measures of the raw or transformed time series data are calculated [1]. Fourier or Wavelet transforms can be applied as transformations [1]. The outcome of the feature selection depends on the calculated descriptive measures, which might not necessarily cover all the spatial and temporal characteristics included in the data, as illustrated in Fig. 1. Applying feature selection directly to the time series has the advantage of being independent of the chosen set of descriptive measures. Only a small number of dimensionality reduction techniques, such as functional PCA as projection technique [14], can directly be applied to time series data.

HMMs are widely used to model the temporal and spatial characteristics of time series and can be directly applied to time series data [15], [16], [17]. We propose a novel feature selection technique based on incorporating the time series as an HMM model and using the F-statistic for feature selection. In comparison to wrapper-based feature selection, this univariate filter technique is independent of the chosen classifier and can be applied to a small number of samples. Wrapper-based approaches are prone to overfitting for a small number of samples in comparison to dimensionality, which is often the case in human movement studies [18].

\section{PROPOSED APPROACH}

The same movement is recorded $m$ times for $n_{i}$ subjects with $i \in\{1, \ldots, c\}$ and $c$ conditions. The conditions or groups can be, e.g., normal and pathological or male versus female movement. A set $\mathcal{K}$ of $k_{\max }$ kinematic parameters is investigated to find a representative subset $\mathcal{K}_{S} \in \mathcal{K}$ with $k_{s}<k_{\max }$. The use of HMMs enables the recordings to have different durations. An individual length of a recording is denoted as $t_{\max }$ and varies between recordings. One recording $\boldsymbol{Y}$ contains the time series $\mathbf{y}_{k}$ of each kinematic parameter $k$ $\mathbf{y}_{k}=\left[\begin{array}{llll}y_{k}(1) & y_{k}(2) & \ldots & y_{k}\left(t_{\max }\right)\end{array}\right]$ and $\boldsymbol{Y}=\left[\begin{array}{lll}\mathbf{y}_{1}^{T} & \ldots \mathbf{y}_{k_{\max }}^{T}\end{array}\right]$.

In the following, feature ranking based on the traditional F-statistic is briefly summarized, the use of HMMs to model time series is described, and the HMM-based extension of the F-statistic to time series is introduced.

\section{A. Traditional F-Statistic}

Descriptive measures $k^{\prime}$ such as minimum, mean, maximum, or range of motion are calculated for each time series $\mathbf{y}_{k}$. The feature mean $x=\bar{k}^{\prime}$ over $m$ repetitions is calculated for each subject and each condition. The overall number of samples $x$ is $n=\sum_{i=1}^{c} n_{i}$. The variance within each group $i$ is denoted as $s_{i}^{2}$, the mean within a group as $\bar{x}_{i}$, the overall mean as $\bar{x}$, and a single sample of group $\mathrm{i}$ and subject $\mathrm{j}$ as $x_{i j}$. 


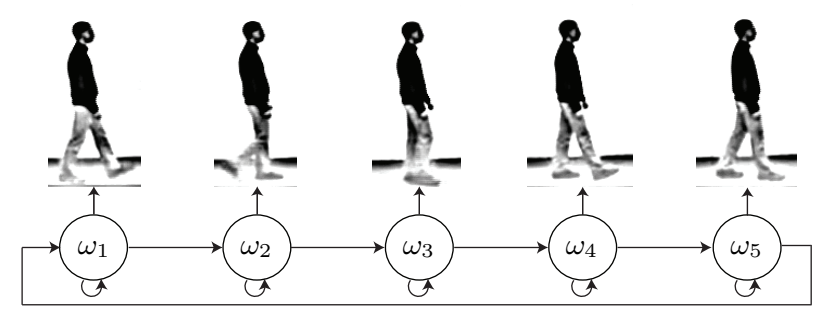

Figure 2. A left-to-right HMM $\left(s_{\max }=5\right)$ transits through the states $\omega_{1}$ to $\omega_{5}$ during a single step. The observations in each state are the kinematic parameters [21, p.115].

The F-statistic computed for a single feature $x=\bar{k}^{\prime}$ is used to compare the variance $S_{W}$ within $c$ groups

$$
S_{W}=\frac{1}{n-c} \sum_{i} s_{i}^{2}\left(n_{i}-1\right)=\frac{1}{n-c} \sum_{i, j}\left(x_{i, j}-\bar{x}_{i}\right)^{2}
$$

to the variance $S_{B}$ between $c$ groups

$$
S_{B}=\frac{1}{c-1} \sum_{i} n_{i}\left(\bar{x}_{i}-\bar{x}\right)^{2}
$$

The larger the F-statistic:

$$
F_{\bar{k}^{\prime}}=\frac{S_{B, \bar{k}^{\prime}}}{S_{W, \bar{k}^{\prime}}}
$$

the better the feature $k^{\prime}$ discriminates between the groups [19]. The F-statistic is also commonly used for hypothesis testing in human movement science.

\section{B. Hidden Markov Model}

HMMs are widely used for time series data [15], [16], [17] and have been applied to action recognition in robotics [20], gait recognition [21], and strategy identification for rehabilitation exercises [4]. An HMM with continuous observations is defined by the parameters $\lambda=\left\{\boldsymbol{A}, \mathcal{N}_{s}\left(\boldsymbol{\mu}_{s}, \boldsymbol{\Sigma}_{s}\right), \boldsymbol{\pi}\right\}$, with the state transition matrix $\boldsymbol{A}$, the observation distribution $\mathcal{N}_{s}$ for each state, and the prior $\boldsymbol{\pi}$. A left-to-right model is used to model the sequential order of $s_{\max }$ hidden states and the observations in each state are the kinematic parameters modeled by a normal distribution $\mathcal{N}_{s}\left(\boldsymbol{\mu}_{s}, \boldsymbol{\Sigma}_{s}\right)$ with mean $\boldsymbol{\mu}_{s}$ and covariance matrix $\boldsymbol{\Sigma}_{s}$. This is illustrated for modeling a single step in Fig. 2 [21, p.115] .

The transition matrix $\boldsymbol{A}$ and the observation distributions $\mathcal{N}_{s}\left(\boldsymbol{\mu}_{s}, \boldsymbol{\Sigma}_{s}\right)$ of the HMM are learned using the Baum-Welch algorithm [15]. It is further assumed that the data is segmented in a way that the HMM starts always in the same state and the prior is $\pi=[1,0, \ldots, 0]$.

Two HMMs are compared using the Kullback-Leibler (KL) divergence as dissimilarity measure $D\left(\lambda_{1}, \lambda_{2}\right)$ [15]. For an oberservation sequence $\boldsymbol{O}^{(2)}$ of length $t_{\max }$ generated by the HMM with the parameters $\lambda_{2}$, the measure is defined as

$$
D_{K L}\left(\lambda_{1}, \lambda_{2}\right)=\frac{1}{t_{\max }}\left[\log P\left(\boldsymbol{O}^{(2)} \mid \lambda_{1}\right)-\log P\left(\boldsymbol{O}^{(2)} \mid \lambda_{2}\right)\right] \text {. }
$$

The measure $D\left(\lambda_{1}, \lambda_{2}\right)$ compares the likelihood $P(\boldsymbol{O} \mid \lambda)$ that the observation sequence $\boldsymbol{O}^{(2)}$ is generated by an HMM with parameters $\lambda_{1}$ or $\lambda_{2}$.
Table I

COMPARISON OF Traditional AND HMM-BASEd TIME SERIES EXTENSION OF THE F-STATISTIC

\begin{tabular}{ll}
\hline Traditional & HMM-based Time Series Extension \\
\hline Sample $k^{\prime}$ & Observation $\boldsymbol{O}=\boldsymbol{y}_{k}=\left[y_{k}(1) \ldots y_{k}\left(t_{\max }\right)\right]$ \\
Mean $x_{i, j}=\bar{k}_{i, j}^{\prime}$ & HMM $\lambda_{i, j}$ \\
Means of each group $\bar{x}_{i}$ & HMM modelling each group $\lambda_{i}$ \\
Overall mean $\bar{x}$ & HMM modelling all sequences $\lambda^{*}$ \\
Distance $\left(x_{i, j}-\bar{x}_{i}\right)^{2}$ & Divergence $D\left(\lambda_{i}, \lambda_{i, j}\right)$ \\
Distance $n_{i}\left(\bar{x}_{i}-\bar{x}\right)^{2}$ & Divergence $D\left(\lambda^{*}, \lambda_{i}\right)$ \\
\hline
\end{tabular}

\section{HMM-Based Extension of the F-Statistic to Time Series}

The concept of the HMM-based extension of the F-statistic to time series is based on Eq. 1-3. The distance measures of the variance $S_{W}$ and $S_{B}$ are formulated as the absolute value of the KL-divergences between HMMs. The F-statistic is computed for each kinematic parameter $k$ individually, so that the observation distributions of the HMMs simplify to a one-dimensional normal distribution $\mathcal{N}_{s}\left(\mu_{s}, \sigma_{s}\right)$ for each state $s$. The transition matrix $A$ and the observation distributions $\mathcal{N}_{s}\left(\mu_{s}, \sigma_{s}\right)$ are learned for a set of HMMs:

- $n_{i} * c$ models $\lambda_{i, j}$ are trained on the $m$ observation sequences for condition $i$ and subject $j$;

- $c$ group-specific models $\lambda_{i}$ are trained on all observation sequences of condition $i$;

- 1 general model $\lambda^{*}$ is trained on all observation sequences.

The variability $D_{W}$ within groups is defined as

$$
\begin{aligned}
D_{W} & =\frac{1}{n-c} \sum_{i, j} D\left(\lambda_{i}, \lambda_{i, j}\right) \quad \text { with } \\
D\left(\lambda_{i}, \lambda_{i, j}\right) & =\frac{1}{m} \sum_{l=1}^{m} \frac{1}{t_{\max , l}} \mid \log P\left(\boldsymbol{O}^{(i, j, l)} \mid \lambda_{i}\right) \\
& -\log P\left(\boldsymbol{O}^{(i, j, l)} \mid \lambda_{i, j}\right) \mid .
\end{aligned}
$$

The larger the variability $D_{W}$ is, the more the models $\lambda_{i, j}$ trained for each subject $j$ differ from the group-specific model $\lambda_{i}$ for each condition $i$. The variability $D_{B}$ between groups is defined as

$$
\begin{aligned}
D_{B} & =\frac{1}{c-1} \sum_{i} D\left(\lambda^{*}, \lambda_{i}\right) \text { with } \\
D\left(\lambda^{*}, \lambda_{i}\right) & =\sum_{j} \frac{1}{m} \sum_{l=1}^{m} \frac{1}{t_{\max , l, j}} \mid \log P\left(\boldsymbol{O}^{(i, j, l)} \mid \lambda^{*}\right) \\
& -\log P\left(\boldsymbol{O}^{(i, j, l)} \mid \lambda_{i}\right) \mid .
\end{aligned}
$$

The larger the variability $D_{B}$ is the more the group-specific models $\lambda_{i}$ differ from the general model $\lambda^{*}$. A comparison of the values calculated for the traditional F-statistic and for the HMM-based extension to time-series is provided in Tab. I.

For each kinematic parameter $k$, the variabilities $D_{B, k}$ and $D_{W, k}$ are calculated. The larger the ratio

$$
F_{k}=\frac{D_{B, k}}{D_{W, k}}
$$


is, the better a kinematic parameter $k$ discriminates among $c$ groups. For $c>2$ conditions, a large value of $F_{K}$ indicates that at least one of the groups is well separable from the other groups. The kinematic parameters $k$ with highest $F_{K}$ values are chosen as features for the feature set $\mathcal{K}_{S}$.

\section{EXPERIMENTS}

The proposed method is applied to a high-dimensional gait database. The F-statistic can be utilized to compare $c \geq 2$ groups. A two-group database is selected to facilitate the illustration and interpretation of the results. The conditions are normal walking and walking after exhaustion. In the following, the dataset and the application of the HMM-based extension of the F-statistic are described. The results are discussed and compared to the traditional F-statistic.

\section{A. Dataset}

The approach is evaluated on a gait database, which contains several strides before exercising and after exhaustion. The database was recorded at the Department of Biomechanics in Sports, Technische Universität München [22].

The gait of 14 male subjects (mean age: $25.3 \pm 2.7$ years; BMI $23.5 \pm 1.9 \mathrm{~kg} / \mathrm{m}^{2}$ ) before and after exhaustion was recorded with a VICON optical tracking system $(240 \mathrm{~Hz})$. 35 passive markers were affixed to the skin of the subjects and the VICON software provides reconstructed joint angles (3 DoF) of the head, neck, shoulder, spine, thorax, pelvis, hip, ankle, and joint angles (1 DoF) of the elbow, knee, ankle, and the foot progress angle (3 DoF). For a 3 Dof joint, e.g., the shoulder, flexion refers to moving the arm forward or backward, abduction refers to lifting the arm sidewards, and rotation refers to rotating the arm around itself. The complete set of $k_{\max }=60$ joint angles is robustly reconstructed for $n_{i}=12$ subjects.

First, each individual's gait was recorded three times and each recording consists of several strides. Then, each subject performed a standardized warm-up program followed by a maximum power test on a rowing ergometer. After 5 minutes rest, a second maximum test was performed and was continued until power decreased below $40 \%$ of individual maximum. Exercise length was about 3-4 minutes and subjects reached a mean heart-rate maximum of $178.5 \pm 9.8 \mathrm{bpm}$. After exhaustion, three consecutive steps were recorded for each subject three times.

The data is segmented into single strides based on the position over time of the marker at the right heel [12]. Three strides are extracted for each condition of each subject and each stride starts and ends when the right foot hits the ground.

\section{B. Extension of the F-Statistic to Time Series}

The HMM parameters are learned using the Baum-Welch algorithm for $12 \cdot 2=24$ models $\lambda_{i, j}$ using $m=3$ observations for each subject $i \in\{1, \ldots, 12\}$ and condition $j \in\{1,2\}$, for 2 group-specific models $\lambda_{i}$ using $12 \cdot 3=36$ sequences of normal walking or walking after exhaustion, and one general model $\lambda^{*}$ using all $12 \cdot 3 \cdot 2=72$ sequences. The number of states are $s_{\max }=15$ and the variance is restricted to $1<\sigma_{s}<$ 50. The observation distributions $\mathcal{N}_{s}\left(\boldsymbol{\mu}_{s}, \boldsymbol{\sigma}_{s}\right)$ for each model are initialized by dividing the time series into $s_{\max }$ bins of equal length and calculating the median and variance of each bin. The median is used instead of the mean to minimize the influence of outliers on estimating initial parameters when only a small number of training samples is available. Furthermore, a training sequence $\boldsymbol{O}$ is viewed as an outlier and discarded from the training set of a model when the probability $P(\boldsymbol{O} \mid \lambda)$ for belonging to this model is lower than the computational accuracy. The frame rate is downsampled to $60 \mathrm{~Hz}$.

The HMM-based F-statistic is computed according to Eq. 7 for each joint angle. This approach does not take into account whether the time series differ in a single descriptive measure such as minimum, mean, maximum, and ROM, or for other descriptive measures, or for a combination of them. It compares the complete time series data of single strides between the conditions, which are normal walking and walking after exhaustion. Fig. 3 illustrates the calculated F-statistics for all joint angles of the left and right side. $F_{k}>2$ is reached for almost half of the joint angles. The F-statistic is highest for left and right head and neck backward tilt, and shoulder abduction of both sides, and additionally for the left shoulder rotation. This observed difference in the kinematics between normal walking and walking after exhaustion is caused by an increased inward posture of the shoulders and head after exhaustion.

The HMM-based F-statistic is symmetric for most joint angles of the left and right side. A symmetry between the left and right side can be expected in particular for the joint angles of the spine, thorax, and pelvis taking human kinematics into account. A large difference between the left and right side is only observed for the shoulder rotation, where two strategies are observed: the time series has either only one positive peak or both one positive and one negative peak. The group-specific HMMs of the left and the right side learn a different strategy, which leads to a large difference in the observed F-statistic.

The learned, group-specific HMMs can be further utilized to investigate the differences between the conditions. The upper plots in Fig. 4 show the time series data for left and right shoulder rotation during single strides. The data for normal walking is illustrated in blue and the data for walking after exhaustion in cyan. A large variation between the trials complicates visual investigation of all sequences. The group-specific HMMs $\lambda_{i}$ provide an average model for each condition shown in the lower plots of Fig. 4 and simplify visual investigation. Visual comparison shows that the group-specific models differ by an offset between $1^{\circ}-2^{\circ}$ during the complete stride.

The average time series are generated in the following way for each group-specific model $\lambda_{i}$ :

1) The expected duration $d_{s}$ is calculated for each state

$$
d_{s}=\frac{1}{1-a_{s s}},
$$

with $a_{s s}$ being the $s$ th diagonal entry of the transition matrix $\boldsymbol{A}_{i}$ [15]. If the expected duration $d_{s}>d_{\max }$ is 

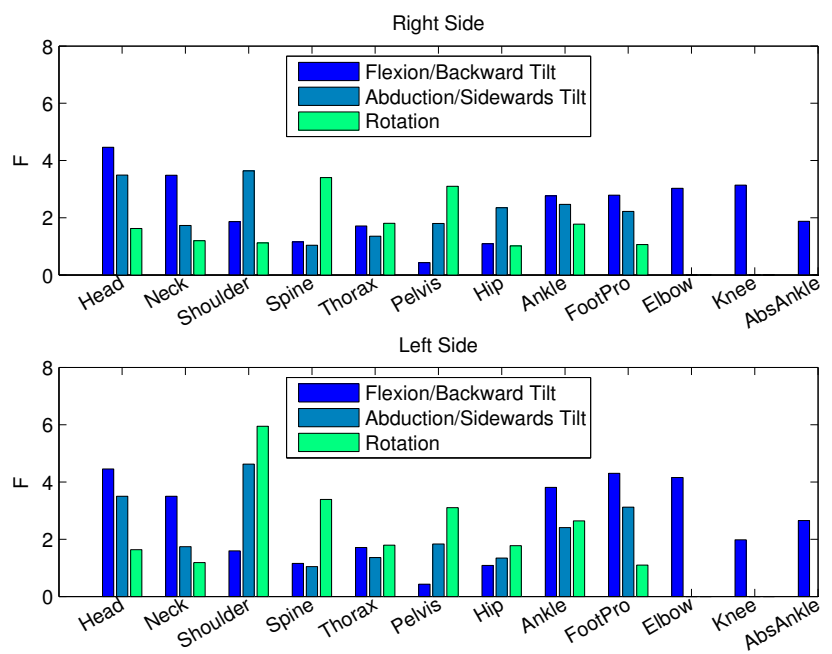

Figure 3. The HMM-based F-statistic is highest for head and neck backward tilt, shoulder abduction, and shoulder rotation. Considering the complete time series of joint angles during a stride, these joint angles differ most between normal walking and walking after exhaustion. This is explained by an increased inward posture of the shoulders and head after exhaustion.
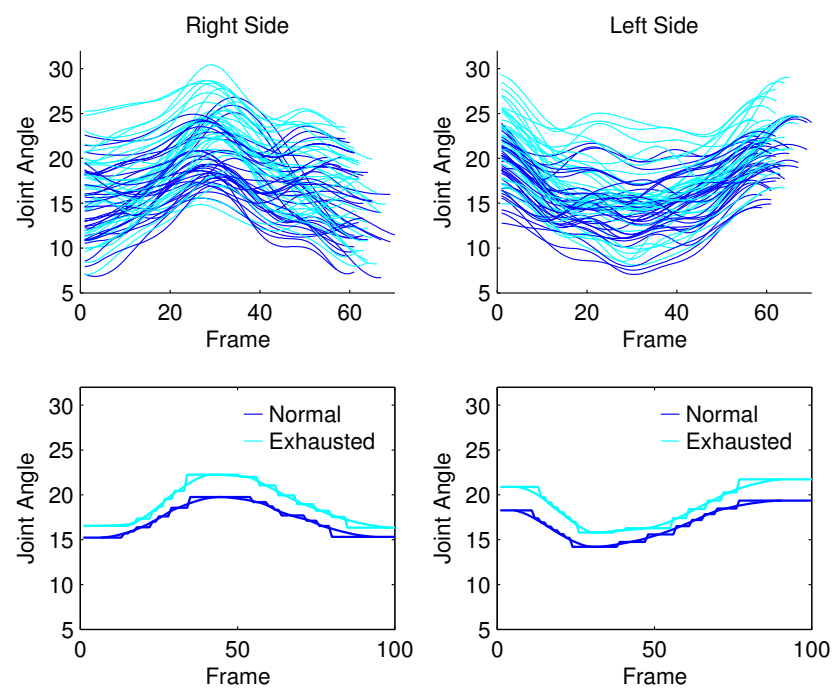

Figure 4. The upper plots show the recorded shoulder abduction angle over time for single strides of normal walking and walking after exhaustion. Large variations between the trials complicate visual analysis. This can be facilitated by generating the average expected time series for each group-specific model $\lambda_{i}$, as illustrated in the lower plots.

larger than a maximum value, it is cut off at $d_{\max }=30$.

2) The mean value $\boldsymbol{\mu}_{s}$ of each state $s$ is plotted during its duration $d_{s}$. This results in a stepped generation of the joint angle over time for each average model $\lambda_{i}$. The generated time series can be further smoothed by applying cubic interpolation to the centers of each state.

\section{Comparison to Traditional F-Statistic}

The highest values for the extended F-statistic are observed for head flexion, neck flexion, and shoulder abduction on the right side and for head flexion, shoulder flexion, and shoulder

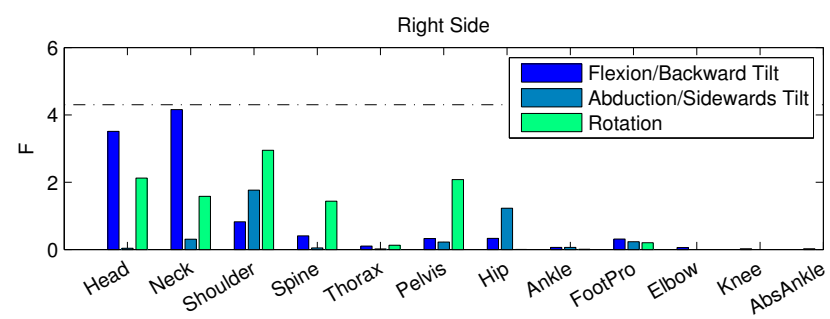

Figure 5. The traditional F-statistic is calculated for ROM of each joint angle during a stride.

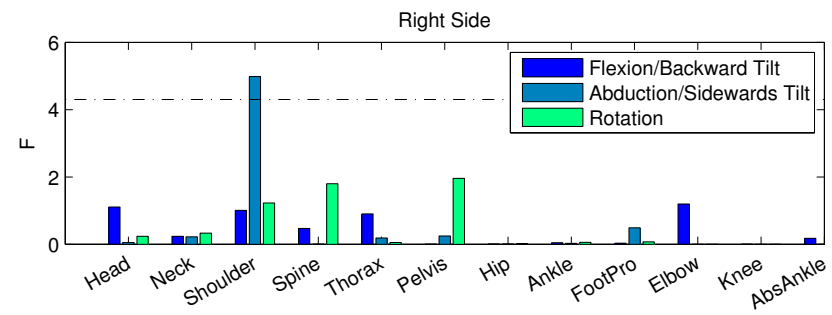

Figure 6. The traditional F-statistic is calculated for the maximum of each joint angle during a stride. The difference between normal walking and walking after exhaustion is statistically significant for shoulder abduction.

abduction on the left side. Furthermore, large values are also observed for the foot progress angle and joint angles of the ankle and the elbow. From these results, it is expected that significant differences between normal and exhausted walking are more likely for descriptive measures of these joint angles.

Descriptive measures can be found by visual analysis of the generated time series of the group-specific HMMs. Fig. 4 illustrates the significance of a spatial offset, such as minimum or maximum, for shoulder abduction. The traditional F-statistic is computed for the minimum, mean, maximum, and ROM for all kinematic parameters to investigate whether values for the traditional F-statistic are statistically significant when the extended F-statistic is large, and whether the traditional Fstatistic is low for kinematic parameters which have a low extended F-statistic. A single descriptive measure can not cover all spatial and temporal variations between two motions. Therefore, it can occur that the extended F-statistic is high but the traditional F-statistic for selected descriptive measures is low.

Fig. 5 shows the results for all kinematic parameters of the right side for the ROM and Fig. 6 for the maximum. When the extended F-statistic is low for a kinematic parameter, also the traditional F-statistic is low. Larger values can be observed for the kinematic parameters where the extended F-statistic is higher. An F-statistic above $F_{1,22, .95}=4.3$ indicates a statistically significant difference between the conditions based on a $5 \%$ significance level (dashed line). This is only the case for the maximum of the left and right shoulder abduction.

Results of the traditional F-statistic depend on the choice of the descriptive measure. In our case, left and right shoulder abduction are important features for the maximum but less important for the ROM. The HMM-based extension of the Fstatistic is independent of the choice of a descriptive measure 
and compares the complete time series without specifying a descriptive measure beforehand.

\section{Discussion \& Conclusion}

An HMM-based extension of the F-statistic is proposed which is directly applied to time series data without the need for descriptive measures such as minimum, mean, or maximum. The utility of the proposed approach is investigated on a gait database. The purpose of the novel technique is to simplify the search for the most discriminative kinematic parameters for biomechanical and clinical applications. The HMM-based extension of the F-statistic compares the relevance of kinematic parameters based on the complete time series and does not require the analyst to specify a descriptive measure beforehand. Results on a gait database show that the technique has the capacity to find the most important kinematic parameters.

The ability to support visual analysis of the temporal data is an important characteristic to achieve clinical relevance for new approaches. This is of great value when specific parameters cannot be easily predefined, for example, during analysis of idiopathic and pathologic gait patterns. The learned group-specific HMMs can be utilized to generate average time series for each kinematic parameter, which can be more easily investigated than the complete set of recorded time series data for each kinematic parameter. These generated time series can be used to select the most suitable descriptive measures.

This approach is related to performing feature selection in the score space [23]. Using the F-statistic for feature selection in the score space has not yet been proposed and investigated.

A limitation of the proposed approach is that it utilizes the $\mathrm{KL}$ divergence as the distance measure. This is a common technique in machine learning to compare HMMs [15]. However, the KL divergence is only a premetric. Furthermore, the exact value of the HMM-based extension of the F-statistic may vary when changing the number of states of the HMM or the sample rate.

The technique is proposed as method before applying ANOVA to select a set of descriptive measures or as a feature selection technique for machine learning. In machine learning, only a few feature selection techniques are applicable to time series data without the calculation of descriptive measures or transformations of the time series data.

\section{ACKNOWLEDGMENT}

The authors acknowledge the support of the Toronto Rehabilitation Institute, which receives funding under the Provincial Rehabilitation Research Program from the Ministry of Health and Long-Term Care in Ontario. The views expressed do not necessarily reflect those of the Ministry. This work was also supported by the Natural Sciences and Engineering Research Council of Canada.

\section{REFERENCES}

[1] R. Best and R. Begg, Computational Intelligence for Movement Science: Neural Networks and Other Emerging Techniques. Idea Group Publishing, 2006, ch. 1: Overview of Movement Analysis and Gait Features, pp. 1-69.
[2] D.Lai, R.Begg, and M. Palaniswami, "Computational intelligence in gait research: A perspective on current applications and future challenges," IEEE Transactions of Information Technology in Biomedicine, vol. 13, no. 5, pp. 678-702, 2009.

[3] S. Wolf, T. Loose, M. Schablowski, L. Döderlein, R. Rupp, H. J. Gerner, G. Bretthauer, and R. Mikut, "Automated feature assessment in instrumented gait analysis," Gait \& Posture, vol. 23, pp. 331-338, 2006.

[4] M. Choudry, T. A. C. Beach, J. P. Callaghan, and D. Kulić, "A stochastic framework for movement strategy identification and analysis," IEEE Trans. Human. Mach. Syst., vol. 43, no. 3, pp. 314-327, 2013.

[5] J. Morrow, A. Jackson, J. Disch, and D. Mood, Measurement and Evaluation in Human Performance. Human Kinetics, 2011.

[6] T. Chau, "A review of analytical techniques for gait data. part 1: fuzzy, statistical and fractal methods," Gait \& Posture, vol. 13, pp. 49-66, 2001.

[7] — , "A review of analytical techniques for gait data. part 2: neural network and wavelet methods," Gait \& Posture, vol. 13, pp. 102-120, 2001.

[8] B. Sundaram, M.Palaniswami, A. Shilton, and R. Begg, Computational Intelligence for Movement Science: Neural Networks and Other Emerging Techniques. Idea Group Publishing, 2006, ch. 4: Computational Intelligence Techniques, p. 1139.

[9] R. Poppe, "Vision-based human motion analysis: An overview," Computer Vision and Image Understanding, vol. 108, pp. 4-18, 2007.

[10] M. Goffredo, I. Bouchrika, J. Carter, and M. Nixon, "Self-calibrating view-invariant gait biometrics," IEEE Trans. Syst., Man, Cybern. B, Cybernetics, vol. 40, no. 4, pp. 997-1008, 2010.

[11] N. Troje, "Decomposing biological motion: A framework for analysis and synthesis of human gait patterns," J VISION, vol. 2, no. 5, 2002.

[12] M. Karg, K. Kühnlenz, and M. Buss, "Recognition of affect based on gait patterns," IEEE Trans. Syst., Man, Cybern. B, Cybernetics, vol. 40, no. 4, pp. 1050-1061, Aug 2010.

[13] Y. Saeys, I. Inza, and P. Larranaga, "A review of feature selection techniques in bioinformatics," Bioinformatics, vol. 23, no. 19, pp. 25072517, 2007.

[14] J. Ramsay and B. Silverman, Applied Functional Data Analysis. Springer, 2002.

[15] L. Rabiner, "A tutorial on hidden Markov models and selected applications in speech recognition," Proc. IEEE, vol. 77, no. 2, pp. 257-286, 1989.

[16] C. Bishop, Pattern Recognition and Machine Learning. Springer, 2006.

[17] R. Duda, P. Hart, and D. Stork, Pattern Classification. Wiley \& Sons, 2001.

[18] R. Kohavi and D. Sommerfield, "Feature subset selection using the wrapper method: Overfitting and dynamic search space topology," in AAAI Int. Conf. Knowledge Discovery and Data Mining, 1995, pp. 192 197.

[19] A. Watkins, R. Scheaffer, and G. Cobb, Statistics from Data to Decision. Wiley \& Sons, 2011.

[20] D. Kulić, W. Takano, and Y. Nakamura, "Incremental learning, clustering and hierarchy formation of whole body motion patterns using adaptive hidden Markov chains," INT J ROBOT RES, vol. 27, no. 7, pp. 761-784, 2008.

[21] M. Karg, "Pattern recognition algorithms for gait analysis with application to affective computing," Ph.D. dissertation, Technische Universität München, 2012.

[22] M. Karg, K. Kühnlenz, M. Buss, W. Seiberl, F. Tusker, M. Schmeelk, and A. Schwirtz, "Expression and automatic recognition of exhaustion in natural walking," in IADIS Int. Conf. Interfaces and Human Computer Interaction (IHCI), 2008.

[23] N. Smith and M. Gales, "Speech recognition using SVMs," in Advances in Neural information Processing Systems, vol. 14, 2001, pp. 11971204. 